to the U.E.M.O. This preparatory work-achieved by the considerable efforts of a few doctors-should prove valuable when Britain enters the Common Market. Not only will the profession's representatives be well versed in the E.E.C.'s medical problems but they will also be widely known among the doctors' leaders in the Six.

It may surprise doctors in Britain accustomed to the ready access their representatives have to government that there is no official link between the Standing Committee of Doctors and the all-important European Commission. ${ }^{3}$ The latter body of nine commissioners-shortly to be joined by Sir Christopher Soames and Mr. George Thomsonhas a staff of 5,000 at its Brussels headquarters, and is the civil service of the community. The commissioners are pledged to independence from their own national governments, and from other particular interests. One of their functions is to prepare proposals for putting to the ultimate authority in the Common Market, the Council of Ministers, which contains one minister from each member government. The Council usually acts only on proposals from the Commission and helping both these top bodies is the Economic and Social Committee, a consultative body over 100 strong, which contains representatives from a wide range of economic and social activities. Thus, for instance, farmers, producers, workers, and merchants sit alongside the professions as well as "representatives of the general interests." The Commission has no official medical advisers of its own, and hence officially the doctors' voice is but one among many. Nevertheless, the existence of the various medical organizations is "acknowledged and they make frequent representations, both written and oral, to the officials of the Commission and to the Economic and Social Committee."3

Of course, views on medical issues can be put forward by the British Government, which has for sometime maintained a close liaison with the B.M.A. on Europe, regularly sending officials from the Department of Health to the Association's E.E.C. Committee. If, as is hoped, civil servants are successfully recruited from Britain to serve in Brussels, then these will no doubt include some staff from the health departments. Hence, valuable expert background knowledge of the N.H.S. should be readily available to the Commission. The regular contacts between the profession and the Government in Britain have been largely a consequence of the character of our centralized State service, which though all-embracing since 1948 really started in 1911. It has no counterpart in the other E.E.C. countries, though the nearest approach to British attitudes to medicine is probably to be found in Holland. In the E.E.C. as a whole, however, the individual medical entrepreneur is still very much in evidence and some of these doctors fear that the arrival of the British N.H.S. may ultimately affect their professional independence.

The various professional problems discussed at the recent London meeting ${ }^{2}$ should be put into the perspective of the Common Market's aim of harmonizing the social services of member countries. ${ }^{3}$ This is a massive long-term reform and Britain's entry will complicate what little progress has been made since 1957. Indeed, Sir Keith Joseph earlier this year emphasized that the Governement "would not rush headlong into arrangements which would have adverse effects on our standards and organization."4 The E.E.C. is primarily an economic union so that the problems of medicine are likely to take a back seat until more weighty international issues have been resolved. Nevertheless, changes in the health arena will probably be agreed eventually, and British doctors will want to know that their views are clearly heard. With the Italians coming to the end of their stint as secretariat to the Standing Committee the B.M.A. seems to be in line to succeed them in $1974,{ }^{5}$ and Britain may be in the thick of medical events during a crucial period of adjustment in the E.E.C.

1 British Medical fournal, 1961, 4, 185.

2 British Medical fournal, 1972, 4, 123.

3 British Medical journal, 1970, 3, 216.

- British Medical fournal Supplement, 1972, 3, 42.

5 British Medical fournal Supplement, 1972, 3, 43.

\section{Violence against Nurses}

The staff of mental hospitals stoically accept the risk of injury as part of their occupation, and perhaps this is why it receives little publicity in the mass media when it occurs. Serious accounts in the scientific journals have also been few, but in the past year or so the results of two wellconstructed investigations have been published which go some way towards making good the gap in our knowledge.

Though not conducted on parallel lines, one investigation complements the other, and both attempt to quantify the acts of violence on staff by patients in the samples studied. The first ${ }^{1}$ is a comprehensive analysis by Bengt Ekblom of such acts in all mental hospitals in Sweden between 1955 and 1964 . Of the 27,773 patients resident in 36 such hospitals $8 \%$ were regarded as dangerous in some degree. Though the author concludes that staff run only a small risk of being seriously injured by patients, it is noteworthy that of 24 major assaults by patients 11 were on staff, one of which on a pupil nurse proved fatal.

The second investigation was in New Zealand. The author, I. D. Robinson, ${ }^{2}$ conducted an inquiry into accidents among nurses at the Oakley Hospital in Auckland between 1962 and 1967. He classifies accidents into three groups. Group I includes accidents due to domestic mishaps such as slipping on wet floors or falling on or down stairs. Group II refers to the ordinary misadventures that may befall any nurse in the routine execution of his or her duties-for example, straining the back when lifting patients out of bed, or sustaining cuts to the hands with broken glass. But of special interest is his group III, in which "the cause is always an aggressive action directed by a patient at a nurse." He goes on to specify 10 methods used by the patient to inflict injury and the region of the body injured, all of them redolent of veterinary rather than human nursing. These include "finger and arm twisting," "kicks to stomach," and "bites to arms or face." Injuries were sustained by a total of 257 nurses (47 men, 210 women), representing a higher incidence than in either groups I or II, which netted 240 and 159 accidents respectively.

Both investigators imply that a shortage of staff increases the risk of aggressive behaviour in patients. The work to be done is of necessity covered by whoever is available. Such expediency may mean the employment of inexperienced and therefore easily rattled staff and of those unfamiliar with the idiosyncrasies of individual patients, who may be inappropriately handled and so provoked to violence. The Swedish study goes into more detail and warns, for example, against the mixing of young aggressive patients with the old and feeble, the outcome no doubt of overcrowding with a consequent lack of opportunity for sensible and logical classification of patients.

These pointers to increased violence have a universal 
applicability, and the remedies seem obvious. But in Britain a special lesson comes out of this work. "Exemptees from legal punishment and patients who have already done something to endanger the safety of others are more apt than other mental hospital patients to be dangerous," Ekblom emphasizes. In Britain an analogus situation obtains in relation to some offenders committed for acts of violence to our conventional mental hospitals under a Hospital Order (Section 60, Mental Health Act, 1959), and to the majority of those committed under a Restriction Order (Section 65 of the same Act), who by definition are dangerous. To continue this practice, inviting disaster, is an act of folly against which we have repeatedly protested in these columns. ${ }^{3-6}$

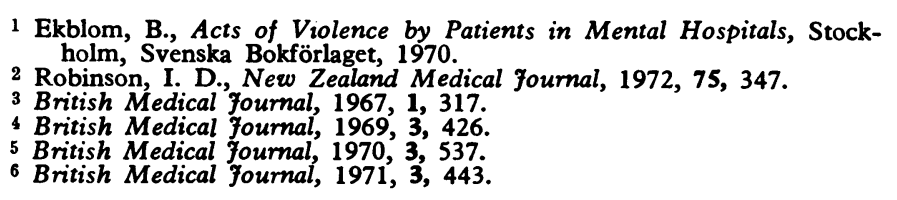

\section{Yellow Nails and Oedema}

It is nearly 10 years since P. D. Samman and W. F. White ${ }^{1}$ first described the combination of yellow nails and lymphoedema. Soon afterwards the association of these with pleural effusions was reported. ${ }^{2}$ Various combinations of these three abnormalities have recently been described by $\mathbf{E}$. Hiller and colleagues in 12 patients at the Mayo Clinic, ${ }^{3}$ and the syndrome seems to be more common than was thought.

The nail changes are usually distinctive and allow the diagnosis to be made even in the absence of other changes. The finger nails are thick, yellowish or greenish in colour, shiny, unduly curved, and grow very slowly. They may show separation from the nail bed, but are usually hard and rather crumbly. When the toe nails are affected the changes may be similar or more nondescript. The nail changes of fungus infections, psoriasis, and of other causes of green nails such as pseudomonas or candida infections are clinically different. 4

The lymphoedema usually affects the lower limb and is often mild. In fact yellow nails are not usually seen with more gross lymphoedema, and swelling of the upper limbs in patients with affected finger nails is exceptional. Since the original report of Samman and White the nail changes have been presumed to be due to defective lymphatic drainage of the nail matrix, but the exact mechanism of this is not yet established. In several of these patients lymphangiography has shown hypoplastic lymphatics, presumably congenital, in affected limbs and even in unaffected regions. This accords with the usually accepted pathogenesis of many cases of lymphoedema-namely, that it is due to a congenital defect of lymph vessels. They can remove extravasated proteins under normal conditions but not when some other pathological process, such as venous stasis, infection, or eczema, increases their load too much.

If lymphatics are defective in fingers and legs it is a short step to postulate that they could be abnormal also in other organs and give rise to pleural effusions. The proof that such effusions are caused in this way is still circumstantial, but some of the Mayo Clinic cases $^{3}$ had been diagnosed as idiopathic after full investigation even for many years. In this series five patients had the full triad of nail, leg, and pleural lesions and three had leg and pleural lesions. Two patients had nail changes and two more nail changes with oedema. These figures, coming from a medical clinic, may give an unduly high incidence of systemic changes in patients presenting with nail changes, and none of Samman and White's cases were noted as having any pulmonary lesions. Hiller and colleagues point out that all their patients also had pulmonary symptoms such as those of bronchiectasis or a persistent cough. There is no evidence that failure of lymphatics predisposes to lung changes, and it seems plausible that these are the inflammatory stimuli which unmask a latent lymphatic defect, just as in the subcutaneous tissues.

The simple message from these studies is that patients with unexplained pleural effusions should have their nails and legs inspected, and patients with the yellow nail syndrome should be questioned for respiratory symptoms.

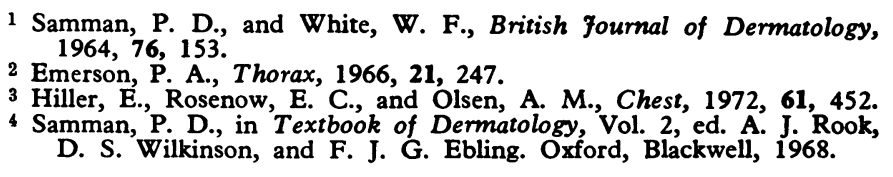

\section{Nobel Prize for Medicine}

Presentday knowledge of the structure of human antibodies is derived from fundamental discoveries made in the late 1950 s and early 1960s. The importance of this work has been recognized by the award last week of the 1971 Nobel prize for physiology or medicine to Professor R. R. Porter, F.R.S., professor of biochemistry at Oxford University, and Dr. Gerald Edelman of the Rockefeller University, New York.

Professor Porter was on the staff of the National Institute for Medical Research when he showed that the immunoglobulin molecule of the rabbit could be split by the enzyme papain. Using advanced biochemical and biophysical methods he succeeded in further subdividing the molecule and was thus able to define its biochemical activity in relation to its structure. His results form the basis of the now famous Porter model of the immunoglobulin.

This model shows that a molecule of immunoglobulin has a basic structure of two "heavy" polypeptide chains joined by disulphide bonds, and that in turn these are joined to two "light" polypeptide chains, which are shorter. Porter showed that the fragments of linked light and heavy chains obtained after treatment with papain were the immunologically active end of the molecule, whereas in this respect the heavy chain fragment was inert.

In recent years six basic classes of immunoglobulins have been discovered-namely, IgG, A, M, D, E, and F-usually at first in patients with specific myelomas. The basic structure of these classes can be related to the original Porter model-for example, IgM is composed of roughly 5 units of IgG. Two major classes of light chains are known, and 6 of heavy chains, and subclasses of both. The Bence Jones protein found in some patients with IgG type of myeloma is related to a production of an excess of light chains by the malignant plasma cells.

Much of our modern knowledge of immunology is related to these discoveries on the components of various immunoglobulins. Future progress in research into antibody production and the vast array of immunological disorders, especially the autoimmune diseases, may come from a more precise understanding of the specific antigen-combining site of the immunoglobulins. Certainly it is difficult to envisage how any progress in recent years would have been made without these fundamental findings and ideas. 